Acta Crystallographica Section D

Biological Crystallography

ISSN 0907-4449

F. Cipriani, ${ }^{a} *$ F. Felisaz, ${ }^{a}$

L. Launer, ${ }^{b}$ J.-S. Aksoy, ${ }^{a}$

H. Caserotto, ${ }^{b}$ S. Cusack,

M. Dallery, ${ }^{c}$ F. di-Chiaro, ${ }^{c}$

M. Guijarro, ${ }^{c}$ J. Huet, ${ }^{a}$

S. Larsen, ${ }^{a}$ Mario Lentini, ${ }^{c}$

J. McCarthy, ' S. McSweeney,

R. Ravelli, ${ }^{a}$ M. Renier, ${ }^{c}$

C. Taffut, ${ }^{\mathrm{c}}$ A. Thompson, ${ }^{\mathrm{a}} \neq$

G. A. Leonard ${ }^{\mathrm{C} *}$ and

M. A. Walsh ${ }^{\text {b* }}$

a EMBL-Grenoble, 6 Rue Jules Horowitz, BP 181, 38042 Grenoble CEDEX 9, France,

bMRC-France (BM14), c/o ESRF, BP 220, 38043 Grenoble CEDEX, France, and ${ }^{\mathrm{C}} \mathrm{ESRF}$, 6 rue Jules Horowitz, BP 220, 38043 Grenoble CEDEX, France

₹ Present address: Synchrotron SOLEIL, L'Orme des Merisiers, Saint-Aubin, BP 48,

91192 Gif-sur-Yvette CEDEX, France.

Correspondence e-mail: cipriani@embl.fr, leonard@esrf.fr, walsh@esrf.fr

\title{
Automation of sample mounting for macromolecular crystallography
}

A standard sample holder and vial for cryocooled macromolecular crystals has been defined for use with robotic sample changers. This SPINE standard sample holder is a modified version, with added features and specifications, of sample holders in common use. In particular, the SPINE standard meets the precision required for automatic sample exchange and includes a cap that is identified by a twodimensional datamatrix code as well as an optional vial. At the ESRF, the sample holder standard is in use with the EMBL/ ESRF/BM14 robotic sample changer (SC3) which is installed on eight beamlines. The SC3 can hold up to 50 crystals stored in five baskets. A datamatrix reader in the SC3 ensures safe management of the sample flow and facilitates fully automatic screening and characterization of samples. Tools for handling and transporting 50 samples in a dry shipping dewar have been developed. In addition to the SC3, the SPINE sample holder is currently compatible with a number of other robotic sample changers.

\section{Introduction}

In recent years, a large amount of effort and resources at synchrotron sites worldwide have gone into providing the user with reliable and stable beamlines that consistently produce high-quality diffraction data for macromolecular crystallography (MX) experiments. In particular, providing the MX user community with rapid access to tuneable beamlines has had a significant impact on the number of structures determined at such facilities (Jiang \& Sweet, 2004). As a result of these efforts it became clear that there was a need to optimize the throughput of the available brilliant light sources by providing tools to search conveniently for the best possible crystal for the experiment in hand. The need to increase sample throughput was further heightened by the launching of structural proteomics initiatives. In these large numbers of structures were envisioned to be determined and thus throughput at all stages of structure determination needed to be optimized.

A major bottleneck encountered by a user at a synchrotron MX beamline has been the time required to get the sample of interest mounted/centred in the X-ray beam and ready for data acquisition. This process is clearly amenable to automation and a number of groups initiated projects to develop methods for automatic sample handling, with the ultimate goal of automating single-crystal diffraction experiments for macromolecules (Cohen et al., 2002; Karain et al., 2002; Pohl et al., 2004; Ohana et al., 2004; Snell et al., 2004; Ueno et al., 2004). Automation of sample handling procedures allows one
Received 2 December 2005 Accepted 4 August 2006
(C) 2006 International Union of Crystallography Printed in Denmark - all rights reserved 
to streamline the data-collection process and reduces the beam-time lost during manual mounting/dismounting of samples. Provision of an intelligent automated mounting system allows identification and tracking of samples throughout the experimental process and paves the way for the automation of screening procedures for identifying the best available sample for data acquisition. Automation of the subsequent data-collection and reduction steps is being pursued at synchrotron sites worldwide, accelerated by the needs of structural proteomics initiatives.

As the development of robotic sample changers from different groups evolved, the need to standardize the sample holder being utilized by these robots was seen as a crucial step forward to encourage their widespread use. The general requirements for a sample holder standard were discussed at a SPINE workshop held in Grenoble in 2002 (see http:// www.spineurope.org for details). In this paper, we describe the development of a SPINE standard sample holder for use with

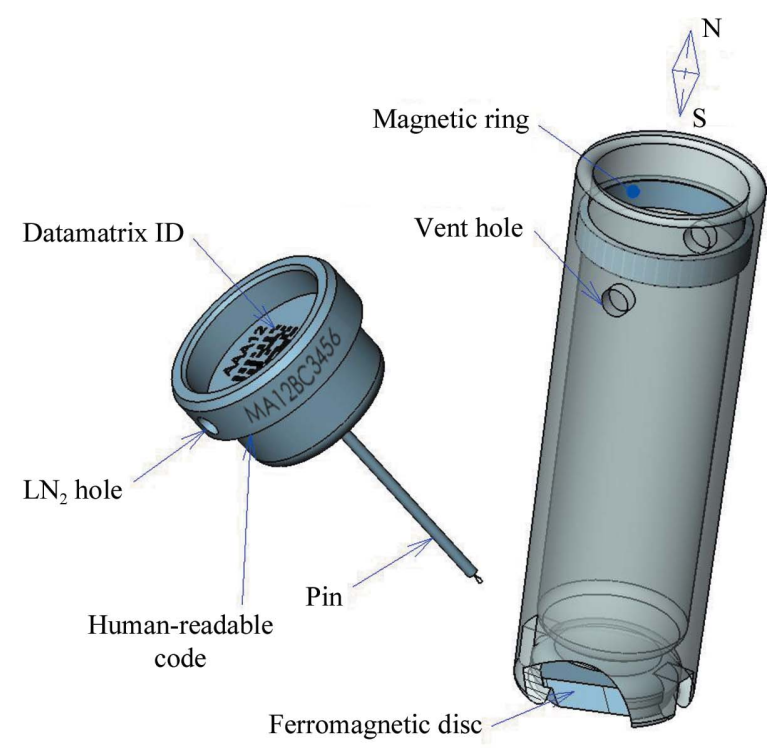

(a)

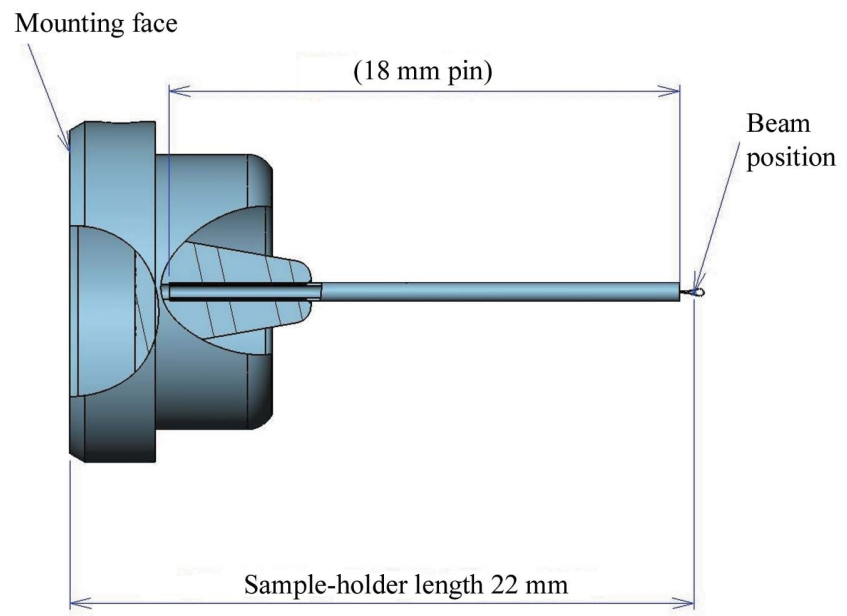

(b)

Figure 1

(a) The SPINE standard sample holder and vial. (b) Definition of the SPINE standard sample holder length and comparison with the Hampton research holder length definition. robotic sample changers and of a robotic sample changer in which this standard has been adopted. In addition to the robot described here, the SPINE standard sample holder is currently compatible with two commercially available robots, namely those from MAR Research (MARCSC system) and Rigaku (ACTOR system), and two academically developed systems, CATS at BM30A (Ohana et al., 2004) and the system developed at the EMBL Hamburg Outstation (Pohl et al., 2004).

\section{The SPINE sample holder standard}

As a result of the SPINE workshop, the development of a standard sample holder for use with robotic sample changers has been driven by the need to ensure that it is machined to the precision needed for robotic handling and the desire that synchrotron users should be able to use several different sample changers without having to resort to specialized holders for each robot.

Another important consideration in the design of the standard was to ensure that it would be adopted within the MX user community; hence, the standard has been designed to inflict as little change as possible on the currently available holders for cryocooled crystals. As with other initiatives (Cohen et al., 2002; Karain et al., 2002; Pohl et al., 2004; Ohana et al., 2004; Snell et al., 2004), it was decided to base the design of the standard on the widespread CrystalCap Magnetic system developed by Hampton Research. Therefore, although the definition of a SPINE standard sample holder is a decisive step for moving smoothly from manual to automatic sample handling, the design and size are nevertheless based on a concept initially developed for manual use. In the future, further optimization of sample throughput will probably require the design of a sample holder specifically optimized for handling by robotic systems.

The SPINE standard (Figs. $1 a$ and $1 b$ ) specifies a sample holder and an associated optional vial. The sample holder is composed of a cap, a pin and a crystal support. The dimensions of the cap allow use with different types of pins and crystal supports including nylon loops, MicroMounts (MiTeGen) and LithoLoops (Molecular Dimensions Ltd). The distance from the crystal to the base of the cap is defined as the 'sampleholder length' and is specified as $22 \mathrm{~mm}$ (Fig. 1b). The surface area of the cap has been optimized to minimize the heating/ drying cycle time that occurs after a sample holder is loaded from a dewar onto the goniometer. Recommendations are also provided on the geometry of the host goniometer magnet/ electromagnet to ensure optimal stability of the sample holder when it is mounted on the goniostat. Full specifications of the SPINE sample holder and vial standard are given in the supplementary material ${ }^{\mathbf{1}}$.

To facilitate management of samples in a high-throughput environment, the SPINE standard incorporates a tencharacter ECC200 datamatrix (DM) code. This allows each

\footnotetext{
${ }^{1}$ Supplementary material has been deposited in the IUCr electronic archive (Reference: GX5085). Details for accessing this material are given at the back of the journal.
} 
sample holder to be uniquely identified and can either be laser-etched or printed onto the base of the cap. Full details of the DM coding are given in the supplementary material. For convenience, an equivalent human-readable code is located on the side of each cap and/or close to the DM code (Fig. 1a). The DM code stores a large number of characters on a small area in a redundant pattern that is tolerant to partial degradation resulting from, for example, ice formation on the cap. The DM codes can be read by commercially available smart-camera/ barcode readers. The system has been tested under liquidnitrogen $\left(\mathrm{LN}_{2}\right)$ conditions and has been shown to work reliably. Reading of the DM codes and entry of associated sample information into an associated Laboratory Information Management System (ISPyB; see Arzt et al., 2005; Beteva et al., 2006) has been streamlined by development of software (Pocket ISPyB) on a personal digital assistant (PDA).

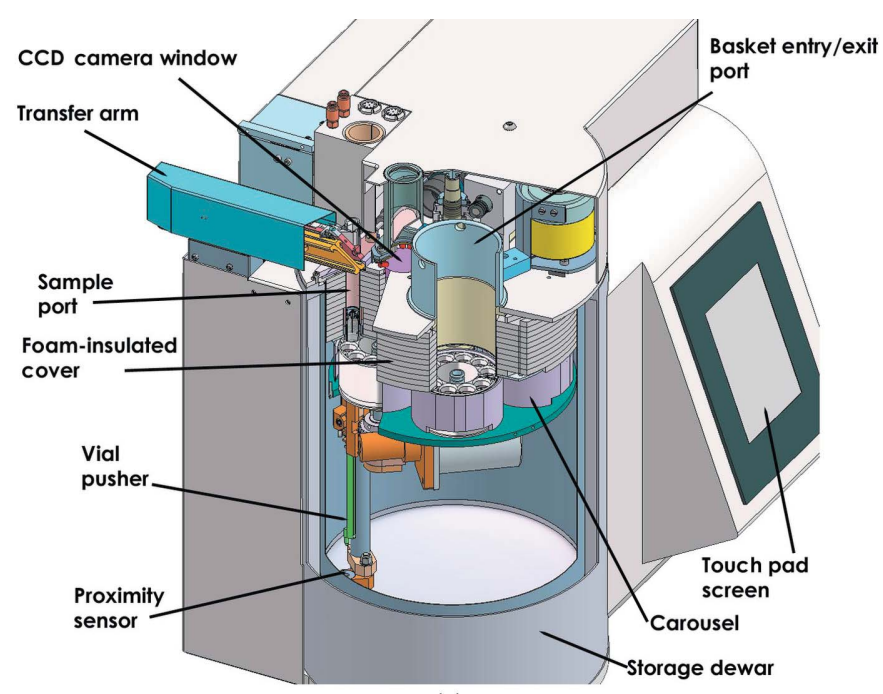

(a)

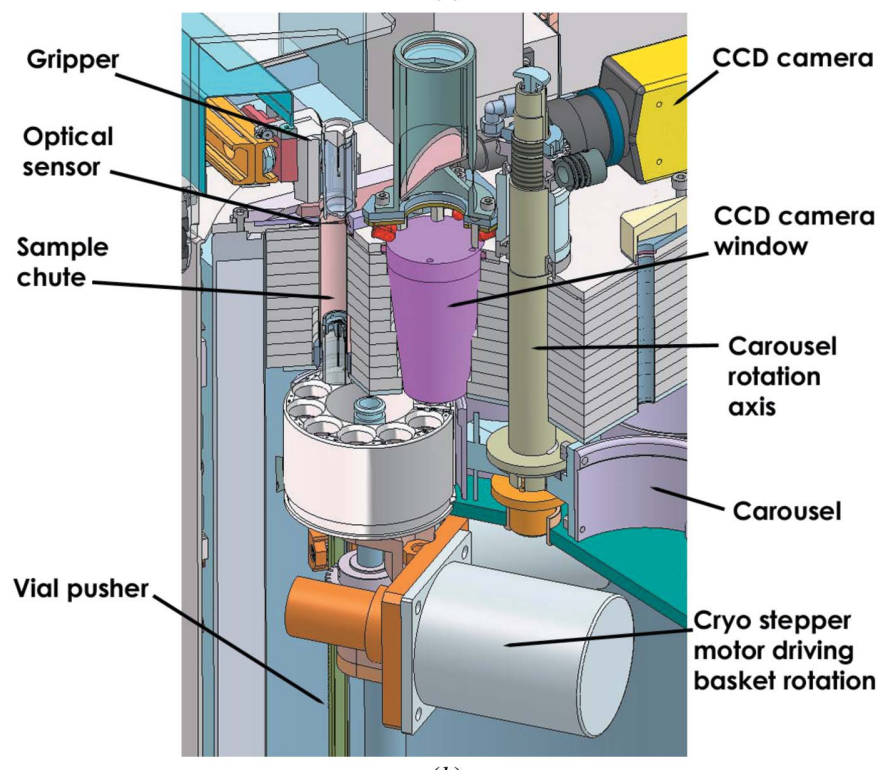

(b)
The vials, developed as an optional extra in the SPINE standard, provide a convenient means of storing and transferring $\mathrm{LN}_{2}$-cooled samples to and from robotic sample changers and remote synchrotron sites for data collection. The vials also allow samples to be transferred to the host goniometer. As in the popular CrystalCap Magnetic system from Hampton Research, a magnetic ring fixes the cap to the vial. However, the SPINE standard also specifies the polarity of the magnetic ring (Fig. 1a). This is essential for reproducible operation in situations where an electromagnet holds the sample holder on the goniometer and where the vial is used to transfer the sample holder. For safety reasons, the SPINE standard also specifies two venting holes located near the top of the vial; these prevent any build up of pressure inside the vial that might occur inside a sealed cap.

Sample holders and/or vials compliant with the SPINE standard are available from Hampton Research (http:// www.hamptonresearch.com), MAR Research (http:// www.marresearch.com) and Molecular Dimensions Ltd (http://www.moleculardimensions.com). For detailed and upto-date information on the SPINE standard and manufacturer information, refer to the SPINE website (http:// www.spineurope.org, protocols menu)

\section{The SC3 automatic sample changer}

\subsection{Background}

In 1999, the ESRF-EMBL Grenoble Outstation Joint Instrumentation Group initiated the development of a robotic sample changer to automatically load and unload cryocooled crystals when using an X-ray goniometer. A first prototype robot (SC1) was tested on a working ESRF beamline (ID29) in 2001. The design of the robot was basic and it used a transfer arm for sample loading from a cryo-storage dewar which could hold 20 samples in a dry environment. These

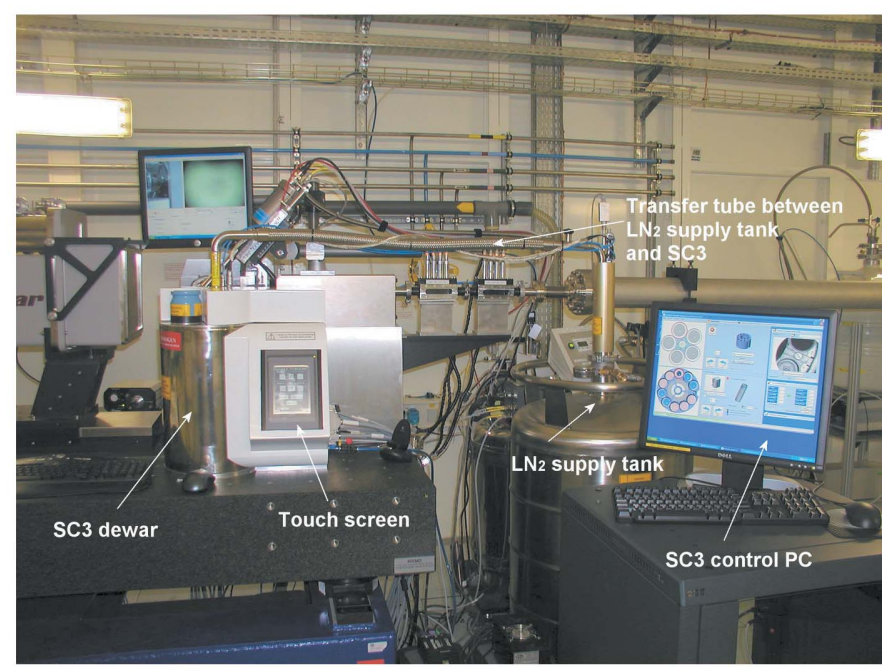

(c)

\section{Figure 2}

(a) Three-dimensional graphical cutaway representation of the main features of the SC3 robotic sample changer. (b) Detailed view of the internal mechanics of the SC3. The detail shows the SC3 basket carousel, associated motor control, location of the sample exit chute, the smart CCD camera and other features of the robot. (c) Overview of the installation of the SC3 at the ESRF. 
samples were mounted using the CrystalCap Magnetic System. The prototype validated the use of a two-axis Cartesian 'transfer arm' to cryogenically transfer a mounted crystal to a goniometer head via the use of a cryovial. It also provided proof of principle that crystals of macromolecules could be kept in stable cryocooled conditions that allowed repeated loading/unloading without any deleterious effects on crystal quality. However, slight random icing problems occurred owing to high humidity in the experimental hutch. These were identified as being the result of water-contaminated air being trapped in the vials during crystal unloads. Therefore, to overcome the problem a 'wet' transfer mechanism was developed for unloading of samples. Briefly, the principle of the wet method is that the robot cryostorage dewar stores the cryovials in an essentially dry cryocooled environment, but to ensure no ice build-up on the samples, the system unloads the crystal into a cryovial filled with $\mathrm{LN}_{2}$ immediately prior to sample unloading.

A second prototype (SC2) including these modifications and piloted using software developed in collaboration with BM14 (MRC, France) was installed and commissioned at beamline ID14-3 of the ESRF in 2003. A production model (SC3) designed to work with samples mounted in the SPINE standard holder and vial has since been produced by the ESRF/EMBL and BM14. Eight units have been installed at the ESRF (beamlines ID14-1, ID14-2, ID14-3, ID14-4, ID29, ID23-1, ID23-2 and BM14) during 2005 and are currently available for operation by users.

\subsection{The SC3 robotic sample changer}

The basic features of the SC3 sample changer are illustrated in Fig. 2. It is composed of the following.

(i) An $\mathrm{LN}_{2}$-storage dewar that holds up to 50 samples stored in five baskets (or pucks) containing ten samples each (see §3.3.1). The baskets are mounted in a five-position carousel via a basket-loading port located on the top of the SC3 dewar.

(ii) An $\mathrm{LN}_{2}$-supply tank and pump.

(iii) A two-axis Cartesian transfer arm with vial gripper for automatic loading/unloading of samples.

(iv) A touch-pad screen for manual control of the SC3. Local control of sample-transfer operations is achieved via the touch screen. Power user (root) options are also available for fine tuning of motor movements, alarm settings and the synchronization of movements that are necessary during installation of a SC3 system.

(v) An electronics crate and a PC. The software running on the PC includes the SC3 user control GUI and device server. The former allows manual and semi-automatic control of the SC3, while the latter allows fully automated sample loading using higher level software such as the $M x C u B e$ data collection GUI at the ESRF (Beteva et al., 2006) that can include intelligent and automated data acquisition via the use of $D N A$ (Leslie et al., 2002).

(vi) A smart CCD-based datamatrix barcode reader.

(vii) An electromagnet with sample holder sensing, mounted on the host goniometer (SmartMagnet). (viii) A pneumatic translation table for supporting the sample cryocooling device.

(ix) User accessories for the handling and transfer of baskets to/from the SC3 and for their transport in a dry shipping dewar.

Once the SC3 has been loaded with baskets containing SPINE sample holders, individual samples can be selected for loading onto the goniometer using either the touch pad SC3 screen, the SC3 control GUI or the beamline-control software $(\mathrm{MxCuBe})$. For a typical load request the basket-selector carousel (Fig. 2b) transfers a selected basket onto the SC3 basket-rotating plate where it is centred using its ferromagnetic base imprint. The basket is maintained magnetically and driven by friction. It is then rotated to position the chosen sample beneath the sample transfer chute. The smart CCD camera ensures proper angular positioning of the baskets by checking the position of the dots engraved on the baskets (Fig. 3). A vertical pusher then lifts the positioned sample holder through the sample chute to the awaiting vial gripper of the transfer arm. A magnet mounted at the end of the vial pusher allows positive handling of the vials (via the ferromagnetic disc set at the bottom of the SPINE vial). A proximity sensor (Fig. 2a) specifically developed to work at $\mathrm{LN}_{2}$ temperatures (see \$3.4) detects the home position of the pusher. The SC3 device server first communicates with the host diffractometer and ensures it is ready to receive a sample. In the case of the experimental setup at ESRF, a number of checks and operations are initiated, i.e. beamstop and sample lighting are moved to avoid collisions and the host goniometer rotation axis is moved to a predefined loading position. During a loading operation the sample holder and vial are translated to the goniometer head, the electromagnet is activated, the sample is loaded and the empty vial is returned to the SC3. In order to avoid collision between the sample holder and the cryocooling head of the host goniometer, the latter is moved, synchronously with loading/unloading operations, a few millimetres back from the sample. The electromagnet that receives the sample is known as a SmartMagnet. This allows the SC3 to sense whether or not a sample is already present on the goniometer head and means that many problematical situations (including the loading of a sample when one is already in place) can be avoided (details are given in \$3.3.2).

When an 'unload' request is received, the empty sample vial is first filled with $\mathrm{LN}_{2}$ within the SC3 dewar. During unloading, the transfer process is synchronized such that the SmartMagnet is demagnetized at the moment the empty vial reaches the goniometer. The sample is then plucked from the goniometer head by the magnetic ring of the vial and both sample holder and vial are returned to the SC3.

\subsection{Detailed description of the SC3 component parts}

3.3.1. The sample-changer dewar. The SC3 sample storage dewar (Figs. $2 a$ and $2 b$ ) is a stainless-steel vacuum-insulated reservoir enclosed by a foam-insulated cover. A number of entry ports are machined in the cover: a $68 \mathrm{~mm}$ diameter port allows loading of the baskets, while a $13 \mathrm{~mm}$ diameter port 
allows the samples holders to exit the SC3 during sample loading. The cover supports the mechanical components used for movement of baskets and sample holders within the SC3 as well as a Cognex smart CCD camera used for viewing the contents of the SC3 dewar. The CCD camera is used both for feedback control of the basket position and to read the DM codes located on both baskets and sample holders. The camera views the contents of the SC3 dewar through a Plexiglas window via a semi-reflective mirror set at $45^{\circ}$ to the camera axis. Uniform reflection-free lighting is essential to reliably read the DM codes. This is achieved by using a system of nine red LEDs embedded on the top of the viewing window which illuminate the field of view of the camera by diffusion of light onto the walls of the viewing-window support.

The dewar is supplied automatically with $\mathrm{LN}_{2}$ by a volumetric pump immersed in the large $\mathrm{LN}_{2}$ supply tank (Fig. $2 c$ ). A constant level of $\mathrm{LN}_{2}$ is maintained such that the bases of the baskets are always steeped in $\mathrm{LN}_{2}$. A dual-level sensor module monitors the $\mathrm{LN}_{2}$ level and passes this to the control software, which drives the $\mathrm{LN}_{2}$ pump accordingly. The supply dewar can hold $120 \mathrm{lof} \mathrm{LN}_{2}$ and is itself refilled automatically. The volumetric pump is connected to the SC3 dewar through a super-insulated pipe and injects, on demand, about $3 \mathrm{ml}$ of $\mathrm{LN}_{2}$ (with a maximum flow rate of approximately $80 \mathrm{ml} \mathrm{min}^{-1}$ or $\left.51 \mathrm{~h}^{-1}\right)$. This on-demand pump operation is essential to fill the empty sample vials with $\mathrm{LN}_{2}$ immediately prior to sample unloading (see \$3.2).

The mechanical components mounted inside the SC3 dewar permit the transfer of the sample holders/vials contained in the baskets to the gripper of the transfer arm. The majority of these components, including the stepper motors, are immersed in $\mathrm{LN}_{2}$. Cleaning of the dewar is thus required every two to four weeks to prevent ice build-up. This operation is facilitated by a 'dryer assistant' installed at the lid position of the basket entry port. A complete cleaning cycle can be performed in about $4 \mathrm{~h}$.

3.3.2. Transfer arm and goniometer SmartMagnet. The compactness of the SC3 sample changer is largely a consequence of the design of its transfer arm (Fig. 4). This two-axis Cartesian robot positions a vial gripper above the exit port on the SC3 to accept a sample holder/vial from the SC3 dewar, translates it towards the goniometer head so that loading/ unloading can take place and finally returns the sample holder/empty vial to a position above the SC3 port from where it is returned to the dewar. The actual loading/unloading of a sample onto the goniometer is achieved using a SmartMagnet whose magnetized state is controlled by the SC3 electronic control rack. During both translational movements a cam is used to rotate the sample holder/empty vial by $90^{\circ}$ so that its orientation is either horizontal ('forward' translation, loading/ unloading onto/from goniometer) or vertical ('backwards' translation, return to SC) at the end of the translation. The gripper holds the vials by means of a small pneumatic jack.

The SmartMagnet (Fig. 5) both holds and detects the presence of sample holders on the host goniometer. The latter function is achieved by measuring the change in inductance of the SmartMagnet coil. Using this system no additional cabling is required for the detection of the sample holder on the goniometer. The control card for the SmartMagnet includes a low-frequency $(300 \mathrm{~Hz})$ oscillator stabilized by quartz in a PLL (phased locked loop) frequency control system and a digital phase-measurement unit. The detection threshold can be changed through an RS232 line. Automatic detection of the sample holder pre-empts manual intervention, as well as providing feedback for possible failure of a sample loading by the SC3.

\subsection{Control electronics}

The electronics of the sample changer are built around a Delta Tau PMAC-CPCI axis-control system (http:// www.deltatau.com). Three main front-end electronic modules are located in the housing of the SC3 itself. The first of these modules monitors the level of $\mathrm{LN}_{2}$ inside the $\mathrm{SC} 3$ dewar which arises from the change on thermal conductivity and measures the temperature change of a silicon diode (heated by a small current) when it is moved from liquid nitrogen to gaseous nitrogen. The second module controls the temperature of the cover of the dewar and the body of the $\mathrm{LN}_{2}$ pump. It also heats the gaseous nitrogen exhaust pipe to avoid ice build-up. Proper temperature control is particularly important in order to avoid condensation forming on the electronic devices installed directly on the cover of the dewar. A dedicated power supply maintains the temperature-control system even when the rest of the electronics are shut down. The third module controls the proximity sensor switch which is used to detect the home position of the vial transfer pusher. This was developed in-house as no commercial switch or sensor was found for operation in $\mathrm{LN}_{2}$. The sensor is a coil that detects the presence of a ferromagnetic object; in this case, the bottom of the vial pusher. The principle used is that described for the SmartMagnet (see \$3.3.2).

A main rack hosts the PMAC controller, the drivers for the two DC and the three stepper motors, the I/O interface logic and the SC3 power supplies. An embedded PMAC program performs the basic operations of the SC3. The touch screen used for manual control of the SC3 is mounted on the front of the SC3 and is connected via a serial line (RS232) to the PMAC CPU.

To ensure safe operation, the SC3 monitors a set of handshake lines that are connected to the electronics of the host goniometer. In addition, two such lines are provided for the monitoring of a chain of safety switches and ensure that collisions between the transfer arm and other equipment such as cryostream heads, back-lighting systems, backstops etc. cannot, under normal circumstances, occur.

\subsection{Control software}

A PC running Windows XP hosts the main control program for the SC3, as well as the GUI and TANGO Device Server required for semi- or fully automatic operation. Three Ethernet ports allow for communication with the control electronics, specifically the PMAC CPU, the Cognex smart CCD camera and the external world (Device Server). 
The GUI provides the user with a graphical view of the hardware and can be used to drive the SC3 remotely from the control hutch. Fully automatic operation of the SC3 is achieved by higher level software such as $M x C u B e$ in concert with DNA (Leslie et al., 2002), which communicates with the SC3 device server. The main page of the GUI is shown in Fig. 6. It has three main tabs: a SC3 operation tab for loading/

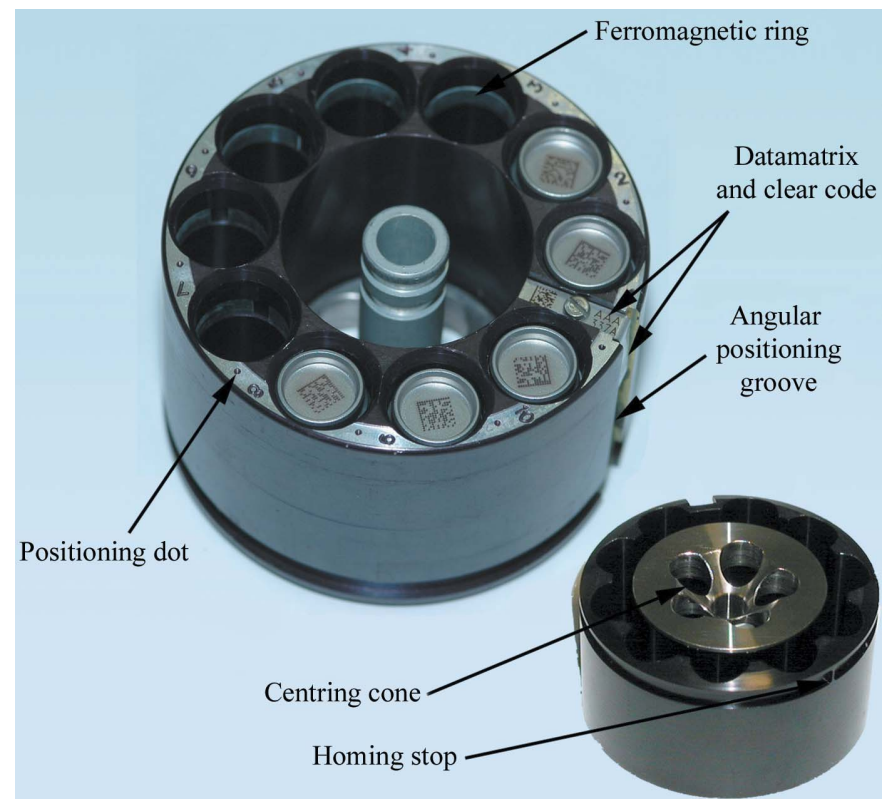

\section{Figure 3}

Overview of the features of the SC3 sample basket or puck which can hold up to ten SPINE sample standards. Five baskets can be stored in the SC3 storage dewar. The baskets are loaded in a carousel inside the SC3 dewar which allows the user to select samples for loading.

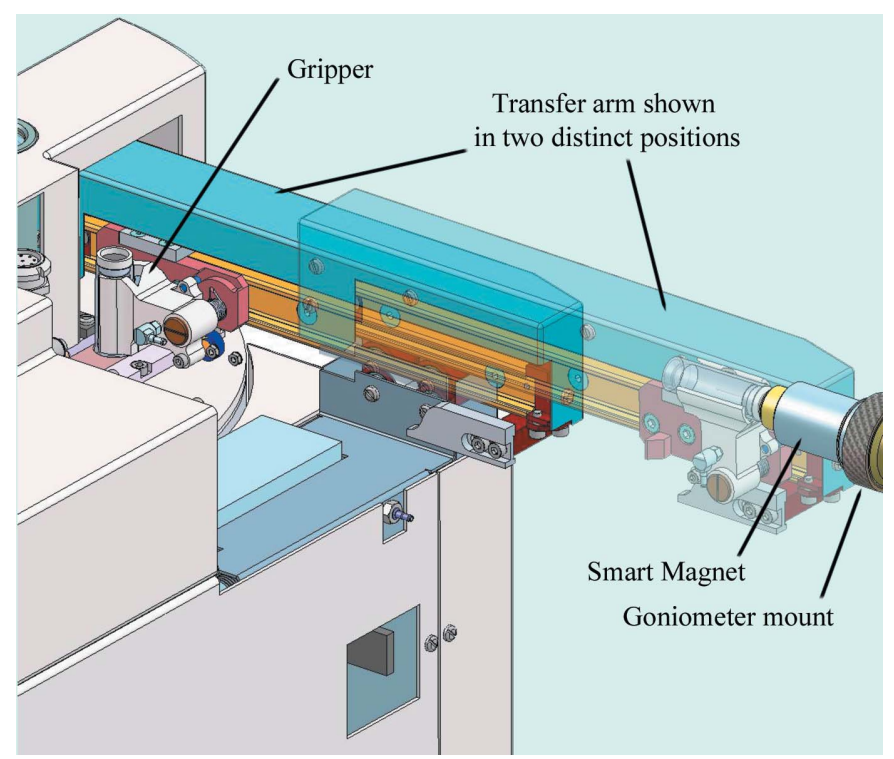

\section{Figure 4}

Three-dimensional graphical detail of the SC3 transfer arm. The transfer arm is shown in its two extreme positions: the initial position where it grabs the SPINE sample vial presented to it from the SC3 sample storage dewar is shown in solid graphics, while the position of the arm when it is presenting the sample holder to the host goniometer is shown in transparent graphics. unloading samples, a sample database tab containing information on all samples mounted in the SC3 and finally a log page which details all SC3 operations and logs errors. In addition, the GUI has several functions implemented to aid alignment and tuning of the SC3 via use of the cognex smart CCD camera.

\subsection{SC3 baskets, accessories and user Starter Kit}

To encourage use of the SC3, a basic Starter Kit has been defined (Fig. 7). This consists of five baskets, a forceps for handling baskets and vials, a vial releaser (to release the vials which are magnetically held in the baskets) and a basket canister allowing storage of the baskets in a dry shipping dewar.

A basket is shown in Fig. 3. It holds up to ten SPINE sample holders mounted with an associated SPINE standard vial. The basket dimensions have been designed to be compatible with a Taylor \& Wharton CX100 dry shipper (http:// www.taylor-wharton.com) or other similar transport dewars such as those supplied by MVE (http://www.sementank.com). Each basket is identified with a unique DM code. The baskets have the following features which aid both manual and automatic handling.

(i) The bases are ferromagnetic, which allows reproducible centring on the SC3 basket-indexing plate.

(ii) A stop is provided on the outer face of the basket for angular homing.

(iii) Ferromagnetic rings are also mounted inside the sample positions. These serve to lock the sample holders in place, thus preventing the empty vials floating in $\mathrm{LN}_{2}$ and limiting the risk of losing a sample during user manipulation of the baskets.

(iv) A series of small dots engraved close to the sample holders slots in conjunction with a smart CCD camera provide a feedback system for control of the basket position.

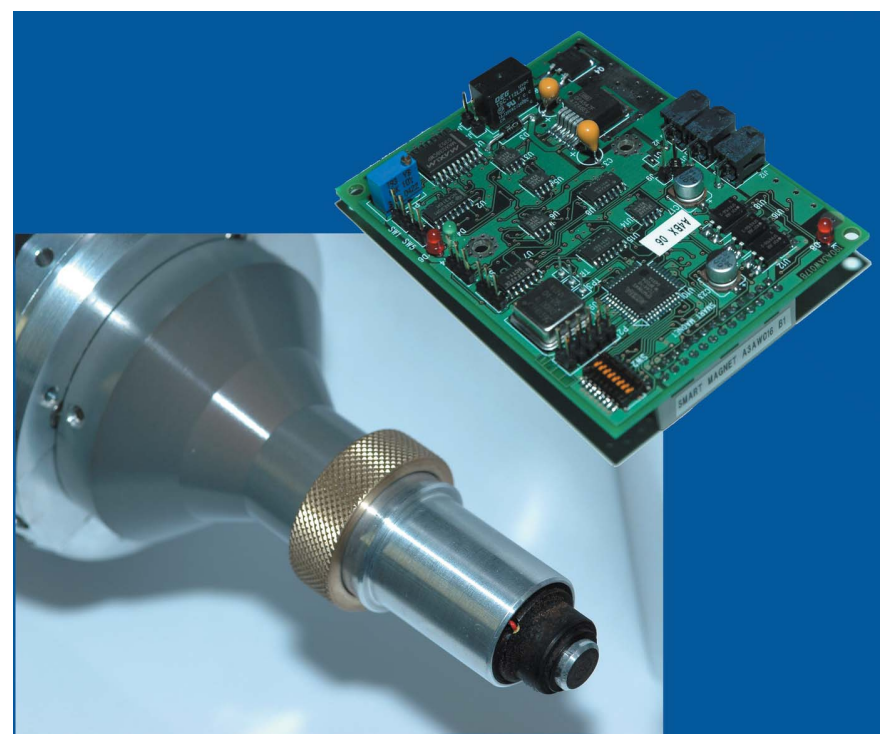

Figure 5

Overview of the SmartMagnet mount in use with the SC3 and its electronic control card. 


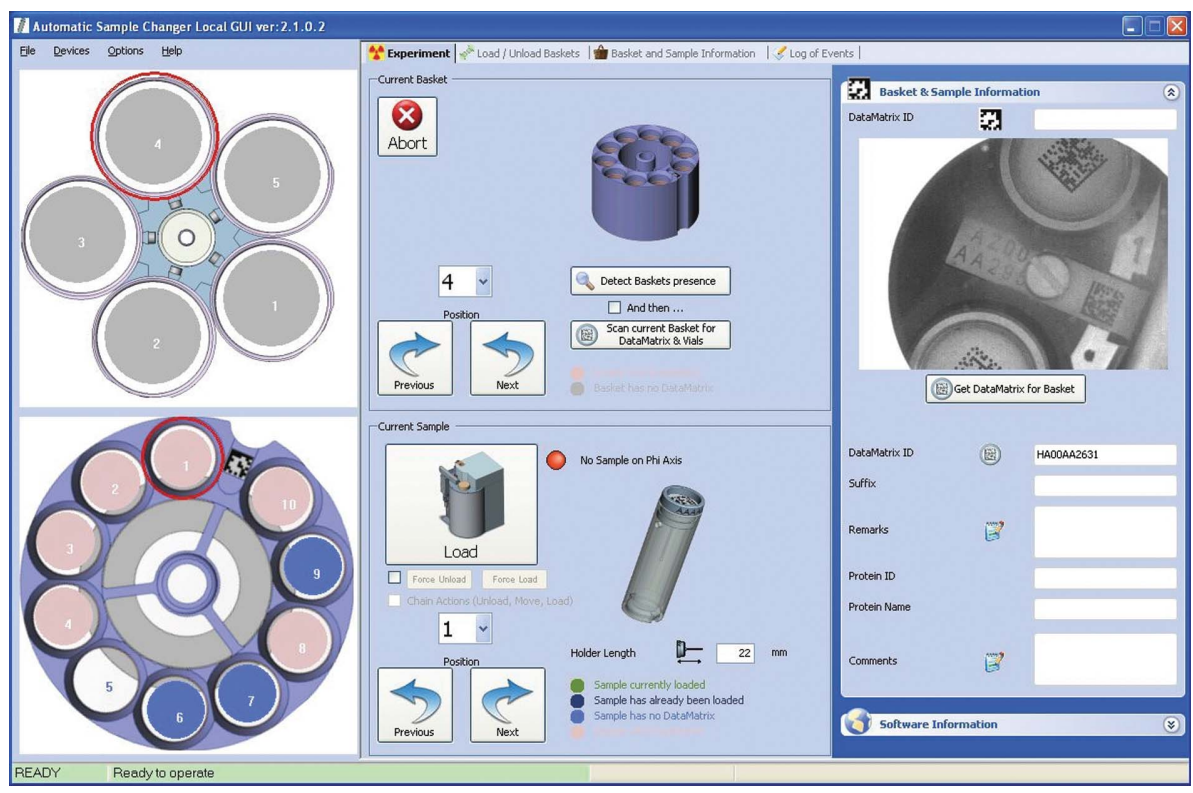

Figure 6

The main page of the SC3 graphical user interface which is used to control the SC3. can then be straightforwardly chosen by multi-axis robots that require a fixed location of the samples in a sample changer.

\subsection{System performance}

The SC3 is currently installed on seven ESRF MX beamlines and the UK CRG BM14. Six of these beamlines are equipped with a minidiffractometer system (MD2M) and two (ID14-3 and BM14) with a microdiffractometer (MD2) (Perrakis et al., 1999). A typical run with the SC3 can be divided up into two phases. The first phase consists of manually loading the SC3 with baskets containing samples. The SC3 then scans the dewar to detect (by reading the DM codes) how many baskets are loaded and how many samples are loaded in each basket. The DM codes associated

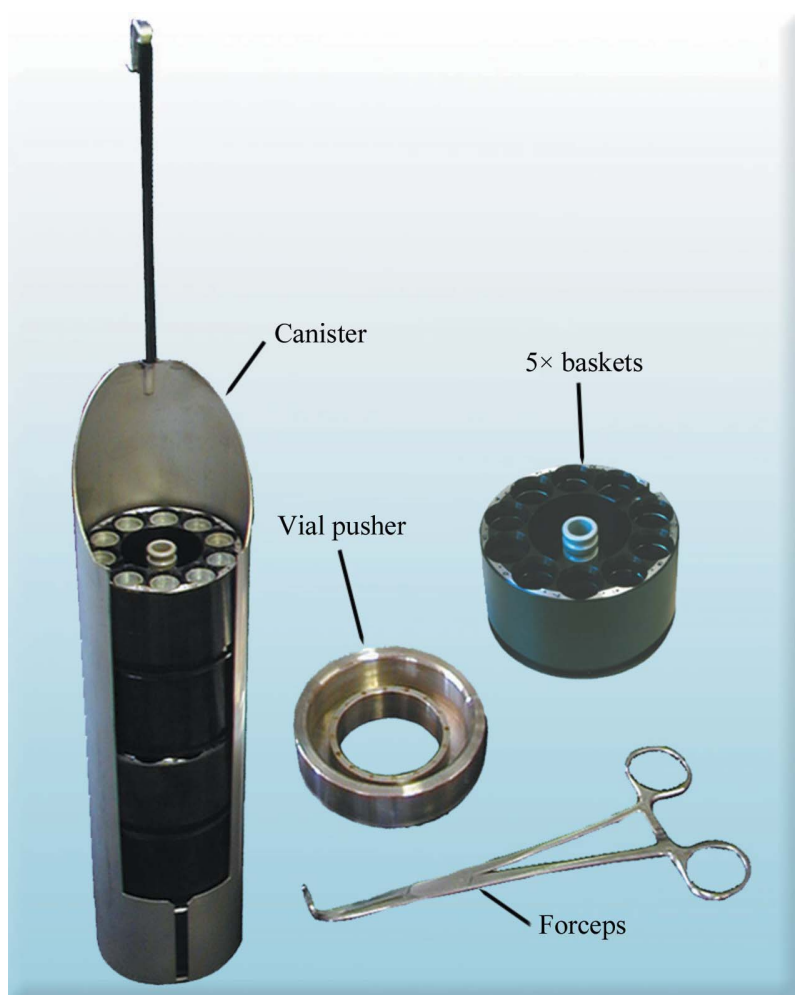

Figure 7

The SC3 Starter Kit which consists of five SC3 baskets, a vial-release tool, a forceps or basket handler and a canister to transfer the baskets with a dry shipper.

Although primarily designed for use with the SC3 robot, the baskets can be used with sample changers based on multi-axis robotic arms. A groove on the cylindrical surface of the basket (see Fig. 3) allows the positioning of the baskets (and hence the samples contained in them) to be fixed. Individual samples with each sample along with its position in the SC3 (i.e. basket number and sample-position numbers) are then displayed in the SC3 GUI.

The second phase is loading of an individual sample. Here, the SC3 communicates with the host diffractometer to ensure the system is ready to accept the loading/unloading of a sample, then sends a request for a sample load to the control software of the diffractometer. This activates removal of the beamstop and backlighting and positions the goniostat in a predefined position for sample loading. The SC3 then positions the selected sample in the load position. The actual loading takes $16 \mathrm{~s}$. Unloading proceeds in a similar fashion and takes approximately $30 \mathrm{~s}$ owing to the additional operation of filling the empty vial with $\mathrm{LN}_{2}$. The cycle time between loading of samples depends on the location of the samples in the SC3. If the next sample is in the same basket, it takes on average $4 \mathrm{~s}$ to position, whereas if the sample selected for loading is in a different basket then an additional $14 \mathrm{~s}$ are required (the time taken to move to the different basket).

At the ESRF, using a modified version of DNA in conjunction with the beamline-control software, the full contents of the SC3 (50 samples) can be screened and characterized automatically (Beteva et al., 2006). In this process each sample is automatically loaded onto the goniometer and centred in the beam using the automated crystal-detection and centring software $C 3 D$ (Lavault et al., 2006). Two diffraction images at $90^{\circ}$ intervals are then taken, autoindexed and used to characterized the crystal, i.e. determine unit-cell parameters, possible space group, diffraction limit and mosaicity. After this, the sample is automatically unloaded. The full process of screening 50 samples takes approximately $150 \mathrm{~min}$ on ESRF beamlines. The time per sample can be broken down as follows: $2 \mathrm{~min}$ to prepare and execute the load/unload of the sample, $50 \mathrm{~s}$ to automatically centre the sample and $6 \mathrm{~s}$ to generate two diffraction images $90^{\circ}$ apart with an exposure 
Table 1

Performance of the eight SC3 robotic sample changers at the ESRF.

\begin{tabular}{lcclll}
\hline & & & $\begin{array}{l}\text { Error } \\
\text { rate } \\
(\%)\end{array}$ & $\begin{array}{l}\text { Days } \\
\text { used } \\
(\% \text { use })\end{array}$ & $\begin{array}{l}\text { Average daily transfer } \\
\text { rate (highest transfer } \\
\text { rate in 24 h) }\end{array}$ \\
\hline ID14-EH1 & 1038 & 9 & 0.9 & $32(71)$ & $32(76)$ \\
ID14-EH2 & 568 & 5 & 0.8 & $22(49)$ & $26(86)$ \\
ID14-EH3 & 658 & 20 & 3.0 & $12(27)$ & $55(158)$ \\
ID14-EH4 & 558 & 32 & 5.7 & $17(38)$ & $33(89)$ \\
ID29 & 937 & 25 & 2.7 & $24(53)$ & $39(122)$ \\
ID23-1 & 1366 & 6 & 0.4 & $27(60)$ & $51(139)$ \\
ID23-2 & 864 & 8 & 0.9 & $18(40)$ & $48(120)$ \\
BM14 & 332 & 2 & 0.6 & $19(42)$ & $18(37)$ \\
Total & 6321 & 107 & 1.7 & $171(48)$ & - \\
\hline
\end{tabular}

$\dagger$ The period reported is from 16 March 2006 to 23 May 2006. This represents 45 days of user beamtime at the ESRF. \$ Errors which required manual intervention to rectify and/or resulted in downtime. These errors were predominately the result of vial jams caused for the most part by use of non-standard SPINE vials or damaged SPINE vials. For beamlines ID14-EH3, ID29 and ID14-EH4 higher error rates are the result of a repeated mechanical jam or failure of the robot during a specific $24 \mathrm{~h}$ period. In the case of ID14-EH4 repeated malfunction of the robot rendered the robot unusable for three separate user groups during the reported period.

time of $1 \mathrm{~s}$. Optimization of the throughput can be achieved by minimizing the dead times of the various components. As an example with the SC3, the sample-unloading phase can be reduced by preparing the unload procedure (filling a vial with $\mathrm{LN}_{2}$ ) while the last $\mathrm{X}$-ray image is being colleted or while the goniometer moves to the transfer position. Other time savings can be made by optimization of readout times of the CCD detector, positioning times for rotation axis etc.

Table 1 summarizes the performance of the eight SC3 systems installed at the ESRF. The period covered is from 16 March to 23 May 2006, which corresponds to $45 \mathrm{~d}$ of beamtime. During this period, approximately $50 \%$ of external user groups made some use of the SC3s. This is very encouraging as SC3 Starter Kits (see \$3.6) only became generally available in February 2006. A proper assessment of the reliability of the SC3s is hard to make at the time of writing. Early experience has highlighted the need to educate users to work with SPINE standard caps and vials, as many 'failures' arose from the introduction of non-SPINE-standard caps and/or vials into the systems. To date, it has not been possible to quantify properly sample-loss rates owing to failure of the SC3 as errors reported relate to any hardware failure which necessitates manual intervention to resolve the problem. Since most of these errors (largely 'vial jams' and 'basket-selector jams') do not result in sample loss, sample loss rates are very much lower.

\section{Conclusions}

We have developed a robotic sample changer (SC3) concomitantly with a sample holder standard that we propose for generic use with robotic sample changers. The driving force for the development of a sample holder standard was to eliminate the burden on the user of having to mount samples in holders specific to each particular robotic sample changer. The development of the standard for European users was initiated via an open-forum SPINE workshop with participation from academic and commercial groups involved in development of automated methods for sample mounting. As a result, the standard is now useable with five independently developed robotic systems.

The SPINE standard incorporates a sample ID which is encoded by an industry standard two-dimensional barcode (Datamatrix ECC200). This provides in principle a generic solution for the tracking of a sample through the structuredetermination process, from target selection to final deposition of the determined structure to the Protein Data Bank.

The SC3 sample-changer robot has been designed to optimize the use of the SPINE-standard sample holders. The SC3 incorporates a smart CCD camera for sample detection and sample identities and histories are presented to the user via the GUI. The SC3 differs from the majority of other samplechanging robots in that it makes use of a cryovial to transfer the mounted sample and does not mimic the use of cryotongs. The approach adopted is less susceptible to ice contamination and provides a seamless transition for sample storage and manual manipulation. The simplicity of the single-axis transfer arm provides a compact design that is easily integrated within a typical macromolecular crystallography end-station.

The SC3 software provides for intelligent and automated use of the robot and can interact and transfer information between higher level software applications such as $D N A$, $I S P y B$ and the EXES system being developed at the ESRF, thus allowing the implementation of a data-collection pipeline (DCP) approach. The DCP implemented at the ESRF (Beteva et al., 2006) is a generic development that also invoves a number of groups dispersed throughout Europe, funded by both national and European research initiatives. The pipeline ensures that information flow to/from ESRF is by an agreed standard. Hence, the management and transfer of acquired data, as well as sample tracking, can be implemented at other sites and provides the user with optimized access to all European synchrotron-based MX facilities.

The SPINE sample holder standard has evolved from input from many groups within the SPINE program (Structural Proteomics In Europe; contract No. QLG2-CT-2002-00988 under the Integrated Programme 'Quality of Life and Management of Living Resources'), but in particular has benefited from extensive input from Ehmke Pohl (SLS) and Arno Lentfer (MAR Research). We also thank Bob Cudney (Hampton Research) for input and support in the development and commercialization of the standard. LL is supported by a BBSRC e-science grant. The successful installation and commissioning of the SC3 was achieved with help from scientists and technical staff at the EMBL, ESRF and BM14; in particular, David Annequin, Hassan Belrhali, Thierry Giraud, Elspeth Gordon, David Hall, Bernard Lavault, Stephanie Monaco, Monique Navizet, Didier Nurizzo, Petra Pernot and Xavier Thibault.

\section{References}

Lavault, B., Ravelli, R. B. G. \& Cipriani, F. (2006). Acta Cryst. D62, doi:10.1107/S0907444906031234. 
Arzt, S. et al. (2005). Prog. Biophys. Mol. Biol. 89, 124-52.

Beteva, A. et al. (2006). Acta Cryst. D62, 1162-1169.

Cohen, A. E., Ellis, P. J., Miller, M. D., Deacon, A. M. \& Phizackerley, R. P. (2002). J. Appl. Cryst. 35, 720-726.

Jiang, J. \& Sweet, R. M. (2004). J. Synchrotron Rad. 11, 319-327.

Karain, W. I., Bourenkov, G. P., Blume, H. \& Bartunik, H. D. (2002). Acta Cryst. D58, 1519-1522.

Leslie, A. G. W., Powell, H. R., Winter, G., Svensson, O., Spruce, D., McSweeney, S., Love, D., Kinder, S., Duke, E. \& Nave, C. (2002). Acta Cryst. D58, 1924-1928.

Ohana, J., Jacquamet, L., Joly, J., Bertoni, A., Taunier, P., Michel, L., Charrault, P., Pirocchi, M., Carpentier, P., Borel, F., Kahn, R. \&
Ferrer, J.-L. (2004). J. Appl. Cryst. 37, 72-77.

Perrakis, A., Cipriani, F., Castagna, J. C., Claustre, L., Burghammer, M., Riekel, C. \& Cusack, S. (1999). Acta Cryst. D55, 17651770.

Pohl, E., Ristau, U., Gehrmann, T., Jahn, D., Robrahn, B., Malthan, D., Dobler, H. \& Hermes, C. (2004). J. Synchrotron Rad. 11, 372377.

Snell, G., Cork, C., Nordmeyer, R., Cornell, E., Meigs, G., Yegian, D., Jaklevic, J., Jin, J., Stevens, R. C. \& Earnest, T. (2004). Structure, 12, 537-545.

Ueno, G., Hirose, R., Ida, K., Kumasaka, T. \& Yamamoto, M. (2004). J. Appl. Cryst. 37, 867-873. 